\title{
PROCEEDINGS OF A SYMPOSIUM
}

\author{
On the \\ NEUROGENIC BLADDER \\ 6TH JUNE I968 \\ at \\ LIVERPOOL UNIVERSITY, ENGLAND
}

\section{GENERAL ASPECTS OF THE NEUROGENIC BLADDER}

\author{
By R. R. HugheS \\ Director of Neurological Studies, University of Liverpool
}

SUBSEQUENT speakers in this Symposium will be dealing with the various types of pathology and certain aspects of the local mechanism of the neurogenic bladder, and hence I intend to confine my remarks largely to the anatomy and physiology of the neurogenic control of micturition, illustrating some of the points with some of my own cases.

During the past 20 years a good deal more information has become available regarding the cerebral control of micturition and it is now apparent that sensory fibres from the bladder reach a conscious level, and efferent fibres pass from the cerebral cortex to control the lower centres for micturition.

There is some evidence that the highest centre for micturition is situated in the upper and posterior part of the frontal lobe (spreading over the inner surface as far as the cingulate gyrus), and in front of the motor cortex. Pathology in this situation, such as a tumour or damage from a leucotomy, may interfere with the conscious control of micturition and lead to urinary incontinence. In some instances, the patient is aware that micturition is imminent, whereas in others conscious appreciation of bladder distension is absent and involuntary micturition occurs unexpectedly. Similarly, an irritable scar in this area of brain may lead to involuntary micturition being the sole or almost the sole manifestation of an epileptiform attack.

In passing it should be noted that an irritable scar in the insula may not only produce epileptic attacks with a visceral accompaniment but may also be associated with a tendency to indecent behaviour and micturition in public. Such patients not uncommonly find themselves in the hands of the police for such behaviour and are referred to psychiatrists for appropriate therapy.

It would seem probable that there is a lower centre for micturition in the pons and it is likely that extrapyramidal fibres in this area normally adjust the bladder tone (and hence the sensitivity of the bladder reflex), in a similar fashion to the adjustment of tone of the voluntary muscles through the muscle spindles. This raises the problem as to whether or not there are muscle spindles or other sensory endings in the bladder muscle. 
In the lateral medullary syndrome frequency of micturition is common and hence it would seem that the efferent fibres from the cerebral hemisphere probably travel in this area of the brain stem; it is of interest that a lesion in this area too will interfere with the sympathetic fibres and produce Horner's syndrome. In the spinal cord the site of the afferent and efferent tracts would now seem to be fairly certain as the result of careful histology of patients who have had bilateral anterolateral cordotomy for intractable pain due to malignant disease. These fibres are present on both sides of the cord about the level of the axis, the motor fibres probably being just anterior to the pyramidal tracts, and the sensory fibres lateral to these in the region of the posterior ends of the lateral spino-thalamic tracts.

Recent work on spinal shock would explain the flaccid bladder which follows immediately on major acute cord damage. The massive disturbance of synapses on the lower motor neurones leads to temporary loss of function of these until function returns once more when the synaptic pattern is re-established weeks later. It would seem likely that the new synaptic pattern is abnormal and hence the spinal bladder is not entirely due to the unmasking of a more primitive physiological reflex but is at least, in part, an abnormal response due to the formation of new synapses.

The most important nerve supply to the bladder is from the sacral cord through the pelvic nerves, which contain both afferent and efferent fibres to the pelvic centre for mircturition. How large a part the sympathetic fibres play in normal micturition is uncertain; so far as one can tell from experimental evidence, the sympathetic supply can be severed without any change in micturition although such damage interferes with normal sexual function.

It is probable too that afferent fibres from the pelvic muscles travelling in the posterior columns can convey some sense of bladder distension due to indirect pressure and hence where a spinal bladder results from bilateral cordotomy the patient may be able to avoid wetting himself as he can take appropriate action when bladder emptying is imminent.

Cauda equina lesions can produce obvious disturbance of bladder function, but the site of the lesion as a rule in this case is fairly obvious on general neurological examination. Recent work from the Mayo Clinic by Love and Emmett suggests that in occasional cases a retropulsed lumbar disc may produce loss of bladder function and chronic urinary retention without any other detectable neurological abnormality. Instances of this type of bladder disturbance in middleaged women are fairly common and hence where there is no evidence of any other pathology to account for the trouble a myelogram should be considered.

\section{PHARMACOLOGIC STUDIES OF THE NATURE OF THE SYMPATHETIC NERVES OF THE URINARY BLADDER}

By Saul Boyarsky, M.D., Peregrina Labay, M.D., Robert Gregg, M.D., and Benton LeVIe M.D.

From Duke University Medical Center, Durham, North Carolina, and the Veterans Administration Hospital, Durham, N.C., Division of Urology, Dept. of Surgery

THE purpose of this presentation is to reconsider the role of the sympathetic nerves of the urinary bladder in the light of the apparent relaxation of bladder tone by Isoproterenol, the beta-adrenergic stimulator. 